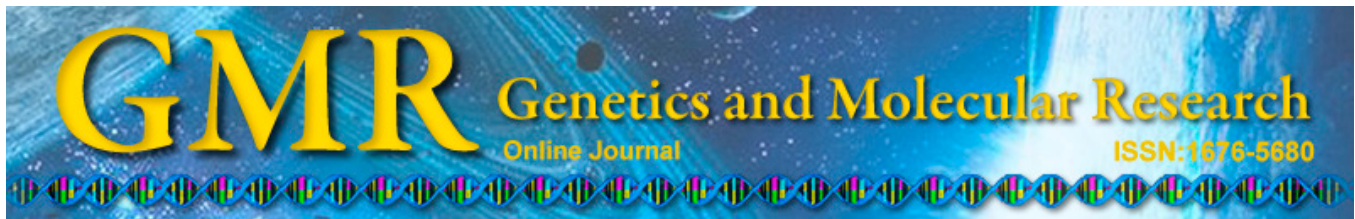

\title{
A new strategy for complete identification of sea buckthorn cultivars by using random amplified polymorphic DNA markers
}

\author{
G. Yang ${ }^{1}$, J. Ding ${ }^{1}$, L.R. Wu ${ }^{1}$, Y.D. Duan ${ }^{1}$, A.Y. Li ${ }^{2}$, J.Y. Shan ${ }^{1}$ and Y.X. Wu ${ }^{1}$ \\ ${ }^{1}$ Berries Research Institute, Heilongjiang Academy of Agricultural Sciences, \\ Suiling, Heilongjiang, China \\ ${ }^{2}$ College of Horticulture, Nanjing Agricultural University, Weigang, Nanjing, \\ China
}

Corresponding author: J.Y. Shan

E-mail: yangg0612@163.com

Genet. Mol. Res. 14 (1): 1836-1845 (2015)

Received May 5, 2014

Accepted September 2, 2014

Published March 13, 2015

DOI http://dx.doi.org/10.4238/2015.March.13.12

\begin{abstract}
DNA fingerprinting is both a popular and important technique with several advantages in plant cultivar identification. However, this technique has not been used widely and efficiently in practical plant identification because the analysis and recording of data generated from fingerprinting and genotyping are tedious and difficult. We developed a novel approach known as a cultivar identification diagram (CID) strategy that uses DNA markers to separate plant individuals in a more efficient, practical, and referable manner. A CID was manually constructed and a polymorphic marker was generated from each polymerase chain reaction for sample separation. In this study, 67 important sea buckthorn cultivars cultivated in China were successfully separated with random amplified polymorphic DNA markers using the CID analysis strategy, with only seven 11-nucleotide primers employed. The utilization of the CID of these 67 sea buckthorn cultivars was verified by identifying 2 randomly chosen groups of cultivars among the 67 cultivars. The main advantages of this
\end{abstract}


identification strategy include fewer primers used and separation of all cultivars using the corresponding primers. This sea buckthorn CID was able to separate any sea buckthorn cultivars among the 67 studied, which is useful for sea buckthorn cultivar identification, cultivar-rightprotection, and for the sea buckthorn nursery industry in China.

Key words: Cultivar identification; New strategy; Sea buckthorn

\section{INTRODUCTION}

Sea buckthorn (Hippophae rhamnoides), a deciduous shrub or bush, belongs to the Elaeagnaceae family. It can provide an effective green protective windbreak and is a useful and durable pioneer plant that prevents soil erosion from wind and water because of its strong ecological adaptability and resistance to extreme conditions such as drought, heat or cold, salinity, and alkalinity. Additionally, its fruits and leaves are rich in vitamin $\mathrm{C}$ and many other bioactive substances with valuable nutritive and medical properties (Lian and Chen, 2000; Jin, 2002). The ecological benefits and economic value of sea buckthorn have led to its development into a major resource in China. Its distribution is widespread, ranging from $2-123^{\circ} \mathrm{E}$ and $27-69^{\circ} \mathrm{N}$, throughout the temperate zone of Europe and Asia (Lian, 1988; Lian and Lian, 1996; Lian and Chen, 2000) and particularly in China, where the germplasm resources and reserves of sea buckthorn are the highest. It is widely distributed in the transition zone of forest-grassland or forest-meadow in the south-Western, north-Western, and northern regions of China (Yu et al., 1993; Chen and Lian, 1994).

Over the last few decades, methods for handling the large repertoire of sea buckthorn germplasm and proper identification of the different cultivars have become necessary. Among the techniques, classical approaches have been used to identify cultivars based on morphological, physiological, and agronomic traits. However, these traits have limitations as they can be easily influenced by the environment and require extensive observation of mature plants. In contrast, molecular markers are uniquely advantageous because they are not affected by the environment and are powerful tools in cultivar characterization. Although DNA-based molecular markers have been utilized in genetic studies, cultivar characterization and identification of sea buckthorn have not been conducted (Sheng et al., 2006). Information regarding genetic diversity levels and the separation of the plant individuals studied have not been reported, and no single report has identified a large number of sea buckthorn cultivars or developed methods that may be useful in future studies. There are currently no methods available that can provide a referable result for the practical utilization of DNA markers in plant cultivar identification. Methods such as DNA fingerprinting have not generated referable information for easy primer determination, and a polymorphic marker for identifying cultivars must be developed. Analysis techniques for DNA banding patterns such as cluster analyses cannot efficiently separate cultivars or species. Employing a strategy for verifying sea buckthorn cultivars in a reliable, easy, referable, and practical manner is crucial for the sea buckthorn nursery and farming industries, cultivar patent protection, and genetic resource conservation and evaluation.

In recent years, various DNA-based markers have been developed and used in genetic diversity, fingerprinting, and cultivar origin studies (Fang et al., 2006; Cheng and Huang, 2009; D’Onofrio et al., 2009; Elidemir and Uzun, 2009; Melgarejo et al., 2009; Papp et al., 2010). Among the DNA-based markers, the random amplified polymorphic DNA (RAPD) (William et 
al., 1990) marker technique is useful for cultivar analysis and has advantages such as its simplicity, efficiency, and non-requirement of any previous sequence information. If optimization of the RAPD technique is conducted by choosing 11-nucleotide (nt) primers and strict screening of the polymerase chain reaction (PCR) annealing temperature for each primer before RAPD is employed in fingerprinting plants, this method can be used for plant cultivar identification. RAPD markers have been widely used in cultivar identification and genetic relationship analysis of a number of fruit species, such as apricot (Ercisli et al., 2009), pomegranate (Hasnaoui et al., 2010), cherry (Demirsoy et al., 2008), pistachio (Javanshah et al., 2007), and strawberry (Wang et al., 2007). Despite their popularity, few DNA markers are available for plant identification.

In this study, we developed a strategy for identifying sea buckthorn cultivars a practical, efficient, recordable, and referable manner, in which a cultivar identification diagram (CID) was constructed manually from RAPD banding patterns. Using our method, we obtained results that differed than those obtained using cluster analysis. The CID, which was used to identify 67 sea buckthorn cultivars, is a valuable service for the sea buckthorn industry in China.

\section{MATERIAL AND METHODS}

\section{Plant materials}

Leaf samples from 67 important sea buckthorn cultivars (Table 1) were collected from the Berries Research Institute, Heilongjiang Academy of Agricultural Sciences, Suiling, Heilongjiang, China. RAPD primers were synthesized by Invitrogen (Carlsbad, CA, USA).

\section{Genomic DNA extraction}

Total genomic DNA of each genotype was extracted from young sea buckthorn leaves using the modified cetyltrimethylammonium bromide (CTAB) method (Murray and Thompson, 1980). Extracted DNA was diluted to a final concentration of $30 \mathrm{ng} / \mu \mathrm{L}$ with $1 \mathrm{X}$ TE buffer and stored at $-40^{\circ} \mathrm{C}$ until use.

\section{RAPD analysis}

For RAPD reactions, 54 random primers, synthesized by Generay Biotechnology (Shanghai, China), were initially tested with a few genotypes and only primers showing products with clear, unambiguous banding patterns for all genotypes tested. These primers were selected for use in genotyping. Eleven-nucleotide RAPD primers were used for screening in this study. To increase the reliability of our results, we used only primers that produced clear, unambiguous banding patterns. Seven primers (Table 2) showing well-resolved and reproducible bands were selected for assaying all genotypes, while the other primers were discarded. Reaction solutions contained $2.0 \mu \mathrm{L} \mathrm{10X}$ buffer, $1.2 \mu \mathrm{L} \mathrm{MgCl}_{2}(25 \mathrm{mM}), 1.6 \mu \mathrm{L}$ dNTPs $(2.5 \mathrm{mM}), 1.6 \mu \mathrm{L}$ primer $(1.0 \mu \mathrm{M}), 0.1 \mu \mathrm{L}$ rTaq Polymerase Dynazyme $(5 \mathrm{U} / \mu \mathrm{L}) 10 \mathrm{X}$ buffer, $\mathrm{MgCl}_{2}, \mathrm{dNTPs}$, and rTaq (TaKaRa, Shiga, Japan), and $1 \mu \mathrm{L}$ genomic DNA, for a total volume of $20 \mu \mathrm{L}$. Amplification reactions were performed based on the standard protocol described by Williams et al. (1990), with minor modifications. PCR was carried out in an Autorisierter Thermocycler (Eppendorf, Hamburg, Germany), programmed as follows: initial 
Table 1. Cultivar name and origin of sea buckthorn used in this experiment.

\begin{tabular}{|c|c|c|c|c|c|}
\hline No. & Cultivar & Origin & No. & Cultivar & Origin \\
\hline 1 & Shen qiu hong & China & 36 & $\mathrm{Za} \mathrm{4-2}$ & China \\
\hline 2 & Shou du & Russia & 37 & HS-19 & China \\
\hline 3 & Fen lan & Finland & 38 & A lie yi & Russia \\
\hline 4 & Xiao la jiao & Russia & 39 & 36 & Russia \\
\hline 5 & Wu ci feng & China & 40 & TF $1-18$ & Russia \\
\hline 6 & Sui 3 & China & 41 & $\mathrm{Za} 56$ & China \\
\hline 7 & HS-4 & China & 42 & TF $1-13$ & Russia \\
\hline 8 & $\mathrm{Za} 4$ & China & 43 & TF2-31 & Russia \\
\hline 9 & You sheng & Russia & 44 & 36 & Russia \\
\hline 10 & Xin e 3 & Russia & 45 & TF2-23 & Russia \\
\hline 11 & Xin e 2 & Russia & 46 & Wu ci xiong & Russia \\
\hline 12 & Xin e 1 & Russia & 47 & 33 & Russia \\
\hline 13 & Chu yi & Russia & 48 & 6 & Russia \\
\hline 14 & HS-23 & China & 49 & 5 & Russia \\
\hline 15 & Cheng se & Russia & 50 & $\delta 4$ & Russia \\
\hline 16 & HS-3 & China & 51 & HS-9 & China \\
\hline 17 & Hun jin & Russia & 52 & $\partial 1$ & Russia \\
\hline 18 & Wu lan ge mu & Mongolia & 53 & Za 2-3 & China \\
\hline 19 & Xiang yang & Russia & 54 & Sui 1 & China \\
\hline 20 & Sui 4 & China & 55 & Lv zhou 4 & China \\
\hline 21 & Sui 2 & China & 56 & Lv zhou 1 & China \\
\hline 22 & A er tai & Russia & 57 & Feng chan & Russia \\
\hline 23 & $\mathrm{Za} 14$ & China & 58 & HS-10 & China \\
\hline 24 & Jin se & Russia & 59 & HS-1 & China \\
\hline 25 & TF2-7 & Russia & 60 & HS-22 & China \\
\hline 26 & TF1-19 & Russia & 61 & HS-12 & China \\
\hline 27 & TF2-27 & Russia & 62 & HS-15 & China \\
\hline 28 & TF2-13 & Russia & 63 & HS-21 & China \\
\hline 29 & TF2-26 & Russia & 64 & HS-20 & China \\
\hline 30 & 2 & Russia & 65 & Ju ren & Russia \\
\hline 31 & $\mathrm{Za} 1-2$ & China & 66 & 37 & Russia \\
\hline 32 & Za 54 & China & 67 & 32 & Russia \\
\hline 34 & Lv zhou 3 & China & 68 & Ka tu ni & Russia \\
\hline 35 & 42 & Russia & & & \\
\hline
\end{tabular}

Obs.: No. 33 is absent.

pre-denaturation step for $5 \mathrm{~min}$ at $94^{\circ} \mathrm{C}$, followed by 42 cycles of a denaturation step for 30 $\mathrm{s}$, an annealing step for $1 \mathrm{~min}$ at annealing temperature (Table 2), and an extension step for $2 \mathrm{~min}$ at $72^{\circ} \mathrm{C}$. Amplification was terminated by a final extension in $72^{\circ} \mathrm{C}$ for $10 \mathrm{~min}$. After amplification, DNA fragments were separated by gel electrophoresis on a $1.3 \%$ agarose $(\mathrm{w} / \mathrm{v})$ (Figure 1) in $1 \mathrm{X} 0.04 \mathrm{M}$ Tris-acetate, $0.001 \mathrm{M}$ EDTA, pH 8.0, buffer at $100 \mathrm{~V}$. The gels were stained with $0.5 \mu \mathrm{g} / \mathrm{mL}$ ethidium bromide and visualized under ultraviolet light. Polymorphic bands among the cultivars were observed in the photographs. Each amplification reaction was repeated at least 3 times to confirm the reproducibility of our results.

Table 2. Seven primers used for the separation of the 67 sea buckthorn genotypes.

\begin{tabular}{llr}
\hline Primer & Nucleotide sequence $\left(5^{\prime}-3^{\prime}\right)$ & Annealing temperature $\left({ }^{\circ} \mathrm{C}\right)$ \\
\hline Y-28 & GTGTGCCCCAT & 43.7 \\
Y-36 & AAGCCTCGTCC & 44.4 \\
Y-48 & ACGACCGACAC & 44.4 \\
Y-42 & AGCGTCCTCCC & 44.4 \\
Y-29 & GTGTGCCCCAG & 43.7 \\
Y-35 & AAGCCTCGTCG & 43.7 \\
Y-41 & AGCGTCCTCCG & 43.7 \\
\hline
\end{tabular}




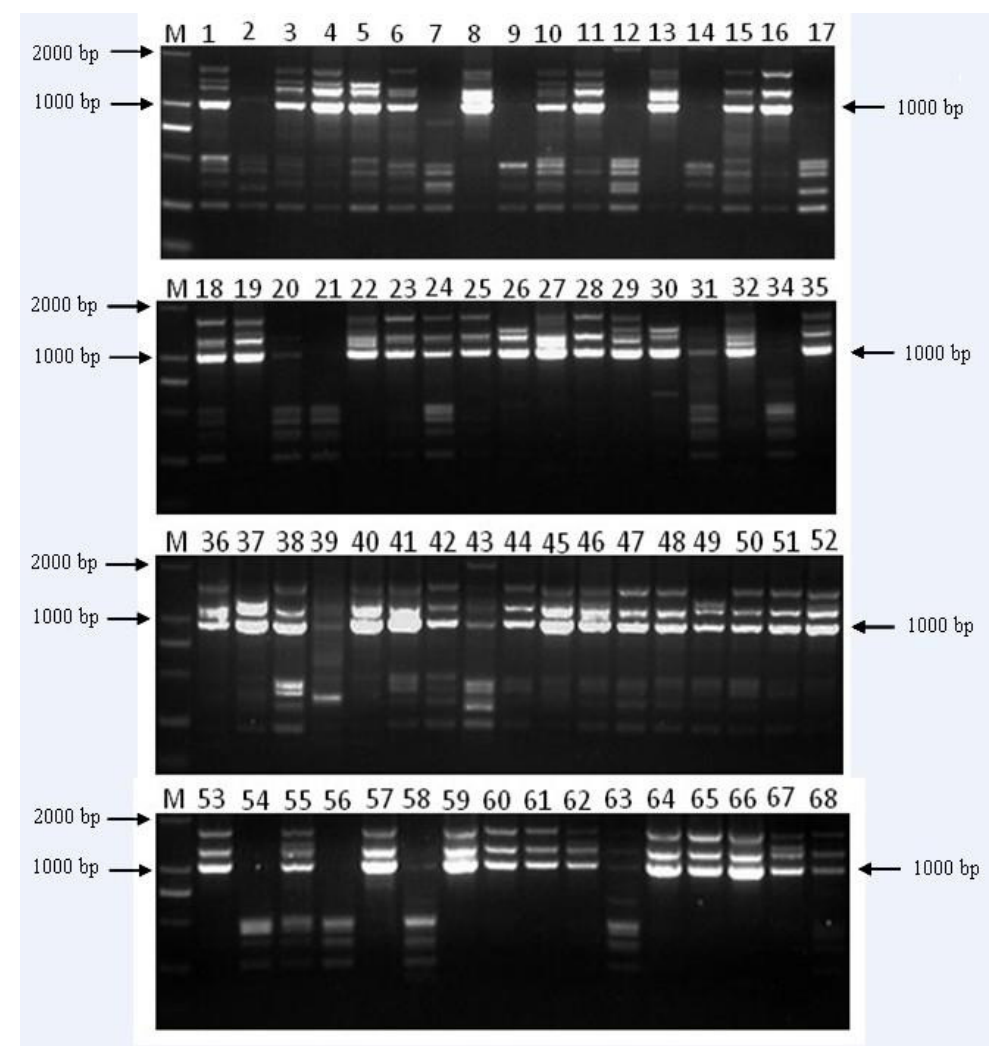

Figure 1. DNA banding patterns of 67 sea buckthorn cultivars amplified using primer Y36. Lane M=DL2000 plus DNA ladders; lanes 1-32 and 34-68= accession numbers of sea buckthorn cultivars listed in Table 1, which are the same as those in the following figures.

\section{Data analysis}

Only clear unambiguous bands in the photographs of gels were scored for cultivar identification. Some cultivars showed a specific band in the fingerprint generated from one primer and could be separated singly, while cultivars sharing the same banding pattern were separated into the same sub-group. Based on this strategy, all sea buckthorn cultivars were separated from one another as more primers were employed.

\section{Utilization and workability of the CID}

Two groups of sea buckthorn cultivars, which were randomly chosen from the interand intra-groups, were used to verify the utilization of the diagram showing the separation of the 67 sea buckthorn cultivars. The 2 groups of cultivars were marked as " $A$ " and "B" and the corresponding primers for separating each group were easily located on the diagram. These cultivars were distinguished accurately and quickly based on the CID, and this method is efficient and can be used with molecular markers to identify other fruit crop cultivars and seed samples of field crops. 


\section{RESULTS}

\section{Cultivar identification}

To establish a stable RAPD system with high reproducibility, longer primers (11 nt) were used and the annealing temperatures for each primer were screened based on the quality and reproducibility of the banding patterns. Primers were randomly screened from a stock of 6011 -nt primers, and once a primer that could produce reproducible polymorphic bands was screened, it was utilized to identify sea buckthorn cultivars. An example is the RAPD pattern obtained using primer Y36, which was the first primer used to amplify the 67 sea buckthorn cultivars examined in this study. The electrophoresis results showed that primer Y36 generated uniform and reproducible band patterns in 14 sea buckthorn cultivars assigned the sample codes $2,7,9,12,14,17,20,21,34,39,54$, 56,58 , and 63 (Table 1). This group of cultivars was easily differentiated from the other 53 cultivars based on the presence or absence of a distinct 1000-bp band; the 67 cultivars were separated into 2 groups. The second primer (Y28) could then separate the 2 groups of cultivars into smaller groups or singly as " 50 ". This separation was conducted sequentially using another 5 primers (Table 2 ) that were screened and chosen to differentiate sea buckthorn cultivars in the sub-groups composed of 2 or more cultivars. Using primer Y41 (Figure 1), all 67 cultivars were completely separated. The total set of 7 primers (Table 2) could identify all 67 sea buckthorn cultivars as shown in Figure 2.

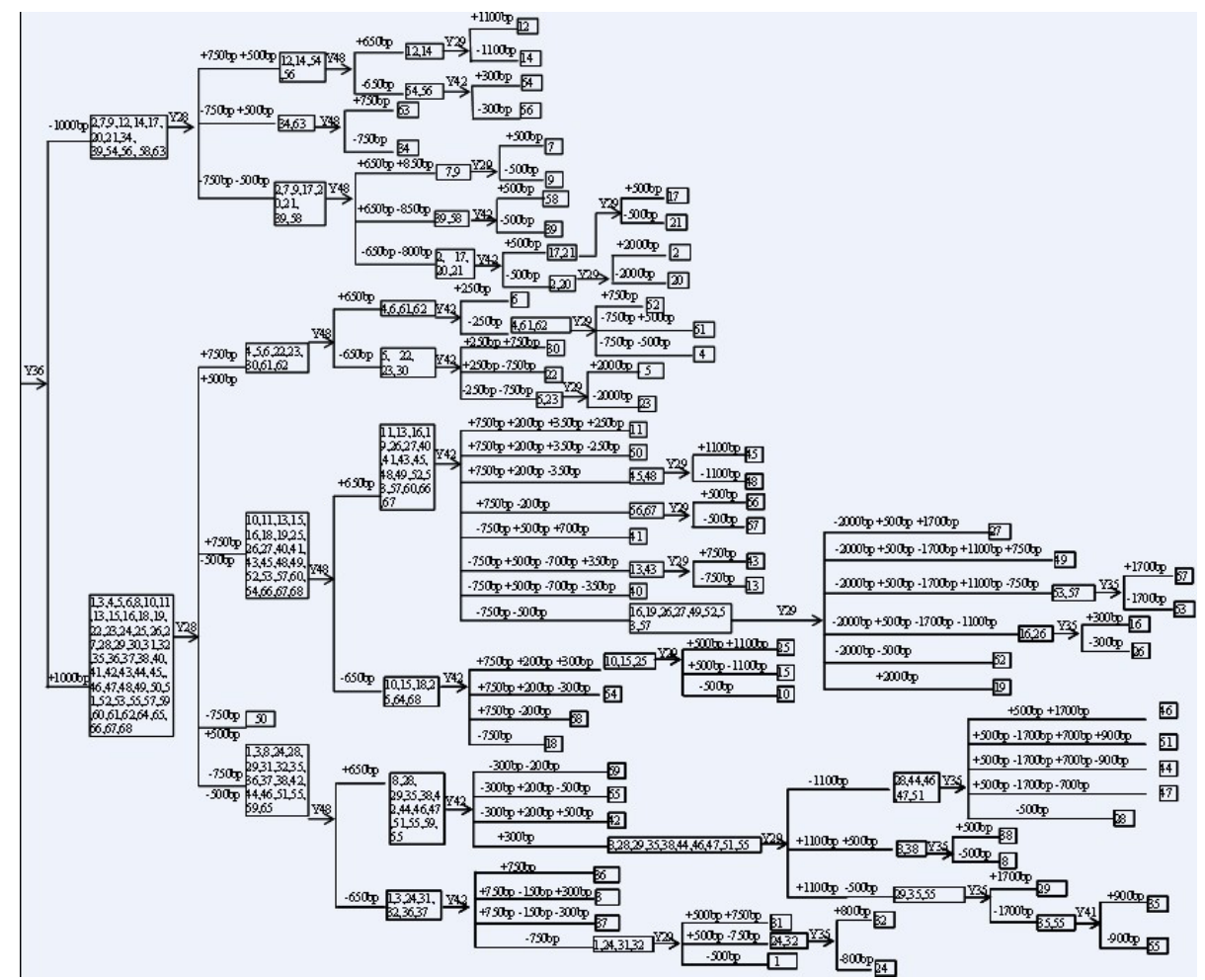

Figure 2. Identification results of sea buckthorn cultivars used by 7 primers and detailed fingerprints. All numbers marked in this chart indicated different size fingerprints, in units of bp; $(+)=$ presence of bands in different sizes; $(-)=$ absence of bands in different sizes. 
Only the clear polymorphic bands generated from each primer were used to differentiate cultivars. The sizes and the presence/absence of polymorphic bands used in the CID are shown in Figure 2, and the CID is very useful for sea buckthorn cultivar identification. At each separation step in the CID, the primer and polymorphic bands amplified by the primer can be considered as a scale-like scheme for separating sea buckthorn cultivars. Therefore, the CID strategy is more useful for sea buckthorn cultivar identification than the currently used cluster analysis.

\section{Test of the utilization and workability of the diagram in cultivar identification}

Although a goal of this study was to develop a technique utilizing RAPD markers to distinguish 67 sea buckthorn cultivars, the larger aim was to generate a referable CID of sea buckthorn cultivars and polymorphic markers that would make it easier to separate and identify sea buckthorn cultivars using the CID. Previous studies focused on the genetic analysis and presence of some phylogenetic trees without referable information for practical plant sample identification. Our findings will benefit the sea buckthorn nursery industry and facilitate cultivar-right-protection.

To identify some of the sea buckthorn cultivars among those examined in this study, the primers can be easily located and the target polymorphic PCR product on the CID can be used for further identification. To confirm this, verification of the effectiveness and efficiency of the sea buckthorn CID was necessary. Three groups of cultivars comprising "12", "13", "14", and " 15 "; " 35 " and " 55 "; " 49 " and " 50 ", which came from the inter- and intra-groups or sub-groups in the CID, were randomly chosen and used for verification. Based on the location of these cultivars in the CID, primers that could be used to separate these 3 groups of cultivars were chosen, including Y36, Y29, Y28, and Y41. The PCR results showed the anticipated banding patterns, and all cultivars in these 3 groups could be disjoined and separated using specific polymorphic bands marked in the CID. The 4 sea buckthorn cultivars in the first group were separated by the PCRs using the primers Y36 and Y29 (Figure 3A). The 1000bp band generated by $\mathrm{Y} 36$ was first separated into 2 groups, from which the group including "12" and "14" could be further disjoined using the primer Y29 based on the 1100-bp band, while another group that included " 13 " and " 15 " was divided by primer Y29 with a band of approximately $1100 \mathrm{bp}$ in size. The group of " 35 " and " 55 " was separated by the 900-bp long polymorphic band derived from primer Y41 (Figure 3B). A band that was approximately 800 bp in length generated using primer Y28 disjoined the group of "49" and "50" (Figure 3C). This validation of separation using randomly chosen groups of cultivars indicates that this sea buckthorn cultivar identification strategy is practicable, workable, effective, and referable, and can be used in the sea buckthorn industry. The data for cultivar separation from the CID can also be placed into a database for future use in silico.

\section{DISCUSSION}

Sea buckthorn (H. rhamnoides) is environmentally important and is a new commercial berry crop. Commercially sea buckthorn is a hardy, multi-purpose plant that produces orange, red, or yellow berries. This plant fixes large amounts of atmospheric nitrogen, and rapidly develops an extensive root system and canopy, quickly covering large areas of soil. These properties make it an ideal candidate for soil and water conservation in extreme or mar- 


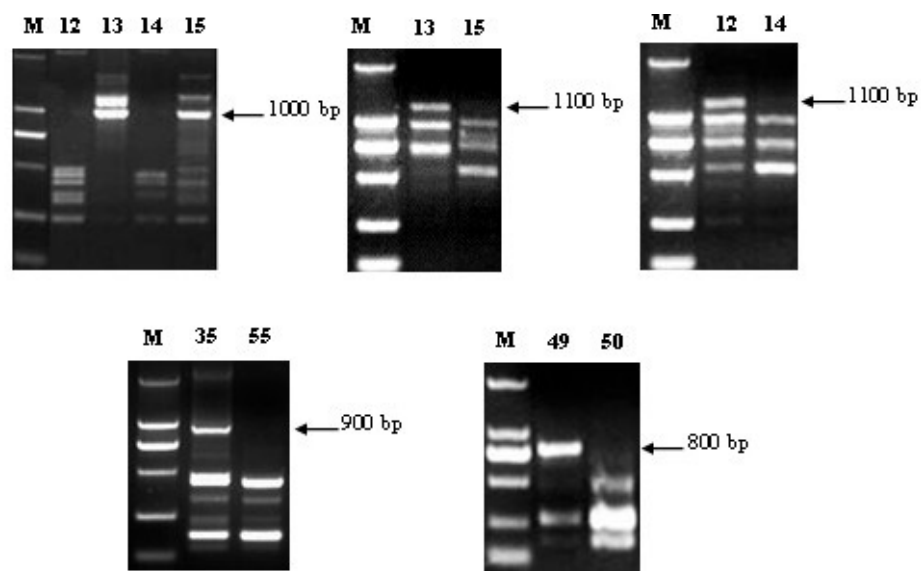

Figure 3. Verification result of several cultivars selected randomly by the corresponding primers. Lane M=DL2000 plus marker; other lanes $=$ accession number of the cultivars used as listed in Table 1.

ginal areas (Ruan and Li, 2005). To improve the development of sea buckthorn research and its related industry, identifying sea buckthorn cultivars and germplasm resources is necessary. Therefore, this study is important for promoting genetic resource conservation and utilization as well as plant variety protection. The development and use of molecular markers in sea buckthorn and related Hippophae species have been previously reported using inter-simple sequence repeats (Jain et al., 2010) and amplified fragment length polymorphism (Ruan and $\mathrm{Li}, 2005)$. However, no efficient strategy for the easy application of DNA markers for sea buckthorn variety identification has been reported. The major goal of this study was not only to use RAPD markers to distinguish the 67 sea buckthorn cultivars based on the principle of DNA fingerprinting, but also to develop a new strategy for properly utilizing DNA markers as a universal strategy for distinguishing other plant and seed samples. An optimized RAPD method maybe can make this strategy more efficient and easier to adapt.

Another result of this strategy is that a readable and referable cultivar identification diagram can be constructed for identifying related plant species in a manner similar to the use of the periodic table of elements, i.e., to provide basic information for each cultivar in a central, universally accessible fashion. This method will be useful in the nursery industry and provide valuable information regarding cultivar-right-protection.

This strategy is efficient for plant identification because few primers and PCRs are needed, and polymorphic bands and DNA fingerprints from various primers can be jointly utilized for further specific identification. In this study, 7 RAPD primers were sufficient to distinguish all 67 sea buckthorn cultivars evaluated. Verification of the accuracy of cultivar identification and the workability of the diagram demonstrated that all groups of cultivars could be distinguished using the specific primers. The present study offers a new method for accurate and reliable identification of sea buckthorn varieties, as well as a theoretical foundation for identifying new cultivars and protecting intellectual property rights (Wang et al., 2009).

This method can be applied to other plant and seed samples, which are important for plant genetic germplasm conservation, cultivar-right-protection, provision of genetically uniform seedlings in production, and the seed industry. Advantages of this method include that only a few primers are necessary and all cultivars can be identified through PCR using 
the corresponding primers found on the diagram. CID information can be transferred to a database in silico and made available to scientists and farmers worldwide. We have initiated additional studies to examine the most important fruit crop cultivars in China for cultivarright-protection, the nursery industry, and genetic resource conservation. We hypothesize that this new method can be used to draw the CIDs for various species, providing information for separating cultivars or varieties as desired.

\section{ACKNOWLEDGMENTS}

Research supported by the National Ministry of Water Conservancy Project "948 Experiment of High Quality Resources of Foreign Seabuckthorn in Black Soil Region of Northeast China" (\#SWCC2012011).

\section{REFERENCES}

Chen XL and Lian YS (1994). The geographical distribution pattern and its formative factors on the genus Hippophae L. Acta Bot. Boreal 14: 105-110.

Cheng Z and Huang H (2009). SSR fingerprinting Chinese peach cultivars and landraces (Prunus persica) and analysis of their genetic relationships. Sci. Horticult. 120: 188-193.

D’Onofrio C, Lorenzis G, Giordani T, Natali L, et al. (2009). Retrotransposon-based molecular markers in grapevine species and cultivars identification and phylogenetic analysis. Acta Horticult. 827: 45-52.

Demirsoy L, Demir T, Demirsoy H, Okumus A, et al. (2008). Identification of some sweet cherry cultivars grown in Amasya by RAPD markers. Acta Horticult. 795: 147-152.

Elidemir AY and Uzun I (2009). Assessment of genetic diversity of some important grape cultivars, rootstocks, and wild grapes in Turkey using RAPD markers. Acta Horticult. 827: 275-278.

Ercisli S, Agar G, Yildirim N, Esitken A, et al. (2009). Identification of apricot cultivars in Turkey (Prunus armeniaca L.) using RAPD markers. Romanian Biotechnol. Letter 14: 4582-4588.

Fang J, Twito T, Zhang Z and Chao CT (2006). Genetic relationships among fruiting-mei (Prunus mume Sieb. et Zucc.) cultivars evaluated with AFLP and SNP markers. Genome 49: 1256-1264.

Hasnaoui N, Messaoud M, Jemni C and Mokhtar T (2010). Molecular polymorphisms in Tunisian pomegranate (Punica granatum L.) as revealed by RAPD fingerprints. Diversity 2: 107-114.

Jain A, Ghangal R, Grover A, Raghuvanshi S, et al. (2010). Development of EST-based new SSR markers in seabuckthorn. Physiol. Mol. Biol. Plants 16: 375-378.

Javanshah A, Tajabadipour A and Mirzaei S (2007). Identification of a new phenotype (Siah Barg) of pistachio (Pistacia vera L.) with shiny-blackish green leaves using RAPD assay. Int. J. Agri. Biol. 9: 307-310.

Jin XJ (2002). Analysis of effective composition of Hippophae rhamnoides L. Chem. Res. Chinese U. 18: 274-276.

Lian YS (1988). New discoveries of the genus Hippophae (Elaeagnaceae). Acta Phytotax. Sin. 26: 235-237.

Lian YS and Lian H (1996). Systematic classification of the genus Hippophae L. Hippophae 9: 15-24.

Lian YS and Chen XL (2000). The regular patterns of distribution on the natural components in plants of the genus Hippophae L. J. Northwest Normal Univ. (Nat. Sci. Edn.) 36: 113-128.

Melgarejo P, Martínez JJ, Hernández F, Martínez R, et al. (2009). Cultivar identification using 18S-28S rDNA intergenic spacer-RFLP in pomegranate (Punica granatum L.). Sci. Horticult. 120: 500-503.

Murray GC and Thompson WF (1980). Rapid isolation of high molecular weight DNA. Nucleic Acids Res. 8: 4321-4325.

Papp N, Szilvássy B, Abrankó L, Szabó T, et al. (2010). Main quality attributes and antioxidants in Hungarian sour cherries: identification of genotypes with enhanced functional properties. Int. J. Food Sci. Tech. 45: 395-402.

Ruan C and Li D (2005). AFLP fingerprinting analysis of some cultivated varieties of sea buckthorn (Hippophae rhamnoides). J. Genet. 84: 311-316.

Sheng HM, An LZ, Chen T, Xu SJ, et al. (2006). Analysis of the genetic diversity and relationships among and within species of Hippophae (Elaeagnaceae) based on RAPD markers. Plant Syst. Evol. 260: 25-37.

Wang C, Fang J, Zhang Z, Cao S, et al. (2009). Brief introduction of fruit cultivar-right-protection in China and its ongoing situation. Chin. Agric. Sci. Bull. 25: 298-302.

Wang ZG, Zhang ZH, Li H, Gao XY, et al. (2007). Identification of strawberry cultivars by RAPD and SCAR markers. 
Acta Horticult. Sin. 34: 591-596.

Williams JG, Kubelik AR, Livak KJ, Rafalski JA, et al. (1990). DNA polymorphisms amplified by arbitrary primers are useful as genetic markers. Nucleic Acids Res. 18: 6531-6535.

Yu ZD, Ao F and Lian YS (1993). The origin, classification, populations and resource of the genus Hippophae L. in China. Hippophae 3: 19-24. 